\title{
Mapeamento e análise espacial de dados com suporte de plataformas web - alternativas para a pesquisa e o ensino remoto na área de geotecnologias
}

Haillany Silva de Souza, Sherlyê Francisco de Carvalho, Elizabeth Maria Feitosa da Rocha de Souza

https://doi.org/10.4322/mp.978-65-991393-8-3.c6

\begin{abstract}
Resumo
A discussão sobre soluções complementares e/ou alternativas para o ensino e a pesquisa utilizando plataformas web cresceu após o surgimento da pandemia da COVID19. Tendo em vista a mudança emergencial para a modalidade de acesso remoto em diferentes instâncias acadêmicas e administrativas, no Brasil e no Mundo, os especialistas precisaram buscar soluções para a continuidade de pesquisas acadêmicas, do ensino e das demais atividades em curso nas escolas, universidades e centros de pesquisa. Diante desse contexto, esse capítulo de livro pretende apresentar alguns exemplos de plataformas, e em especial aquelas que utilizam modelos de análise espacial para um grande volume de dados (como a plataforma Google Earth Engine), que configuram os modelos chamados "Big Data" e Cloud Computing. A análise do espaço, e das ações de agentes transformadores deste espaço, deve acompanhar a evolução tecnológica que se apresenta, sobretudo na área de geoinformação. A abordagem tradicional, que se baseia no uso de ferramentas desktop, vem sendo gradualmente complementada com o uso de plataformas web, seja para um simples armazenamento de dados ou mesmo para o processamento com o uso de ferramentas de análise espacial. Nesse contexto, a computação em nuvem, se apresenta como um modelo atual para a geração de mapeamentos, configurando um novo paradigma para a construção de aplicações em geoprocessamento especialmente na modalidade remota. Dessa forma, o presente trabalho aponta algumas plataformas de computação na nuvem e mapeamento web como importantes mecanismos de fomento às demandas da área de Geografia. A presente pesquisa agradece o apoio e financiamento da FAPERJ por meio do Edital JCE - 2018 - Jovem cientista do Estado
\end{abstract}

Palavras-chave: big data; clouding computing; geoinformação; plataformas web.

\section{Introdução}

As transformações tecnológicas que o mundo vem sofrendo foram impulsionadas pelas mudanças ocasionadas pela pandemia de Covid-19, que demandaram alternativas para a continuidade de pesquisas e o ensino na modalidade remota. Desta forma, as plataformas web se mostram bastante favoráveis como potenciais ferramentas de estudo, visto que elas permitem a integração e a análise de dados frente ao isolamento que a pandemia nos exige. A modalidade tradicional e uso das plataformas de SIG (Sistemas de Informação Geográfica) usam softwares com 
capacidade para armazenar, processar, analisar e representar as informações sobre determinado espaço geográfico, integrando dados em redes físicas ou desktops. Com o crescente volume de dados produzidos pelas instituições e usuários em todo o mundo, associado ao aumento da capacidade de processamento pelos hardwares, observa-se o uso de dados em plataformas WEB e com dados na nuvem, se destacando de forma marcante. Nessa circunstância, as plataformas web se apresentam ricas em possibilidades, visto que além de complementar às plataformas tradicionais, a única ação necessária para fazer uso das mesmas é o acesso estável à internet. A adoção dessas plataformas web para a análise e integração de dados espaciais permitem, via acesso remoto, a continuidade das pesquisas acadêmicas, do ensino e das demais atividades em curso por escolas, universidades e centros de pesquisas em todo o mundo. Dentre as vantagens pode-se citar ainda a integração de pessoas e pesquisas, a divulgação acelerada de resultados de pesquisas entre outras vantagens. Outro grande atrativo para o seu uso, é que estas plataformas utilizam modelos de análise espacial que possuem capacidade para processar um grande volume de dados, cujos modelos são popularmente conhecidos como Big Data e Cloud Computing.

Segundo Amaral [1], Big Data é o fenômeno em que dados são produzidos em vários formatos e armazenamento por uma grande quantidade de dispositivos e equipamentos. Essa evolução tecnológica se deu pelo barateamento dos insumos de tecnologia, memórias, unidades de armazenamento, bem como desenvolvimentos e melhorias em miniaturização, capacidade de processamento.

A computação em nuvem por sua vez ou Cloud Computing, surge como um novo paradigma que apresenta a computação como um serviço de utilidade, apresentando vantagens como: o fornecimento rápido e flexível de poder computacional; a capacidade de computação combinada para melhor utilizar e compartilhar recursos; 0 acesso de banda larga para comunicação rápida; o acesso sob demanda para a computação como um serviço utilitário; não há a necessidade de pagar uma grande quantidade de custos iniciais como recursos de computação tradicionais. Para Amaral [1], a arquitetura orientada a serviços é adotada na computação em nuvem e permite "tudo como serviço", incluindo as mais conhecidas infraestruturas como: O serviço (laaS), a plataforma como serviço (PaaS) e o software como serviço (SaaS). Recentemente observa-se ainda o crescimento do Banco de dados como serviço (DBaaS ou DaaS), e da rede como serviço (NaaS). O Big Data traz inúmeros desafios, mas em conjunto com Cloud Computing tentam encontrar maneiras de minimizar as dificuldades de se trabalhar com grande velocidade e volume de dados e vem provando ser uma solução eficiente para análise, processamento e armazenamento desses dados.

A Figura 1 destaca a perspectiva de integração de dados e circulação de informações produzidas no âmbito do Big Data e Cloud Computing. Segundo Yang et al. [2] o Cloud Computing surge como um novo paradigma para fornecer computação como um serviço utilitário para atender às diferentes necessidades de processamento com a) serviços sob demanda, b) recursos agrupados, c) elasticidade, d) acesso de banda larga e e) serviços medidos. A utilidade de fornecer capacidade de computação promove uma solução potencial para a transformação dos 4 Vs do Big Data no $5^{\text {o }}$ (valor). 


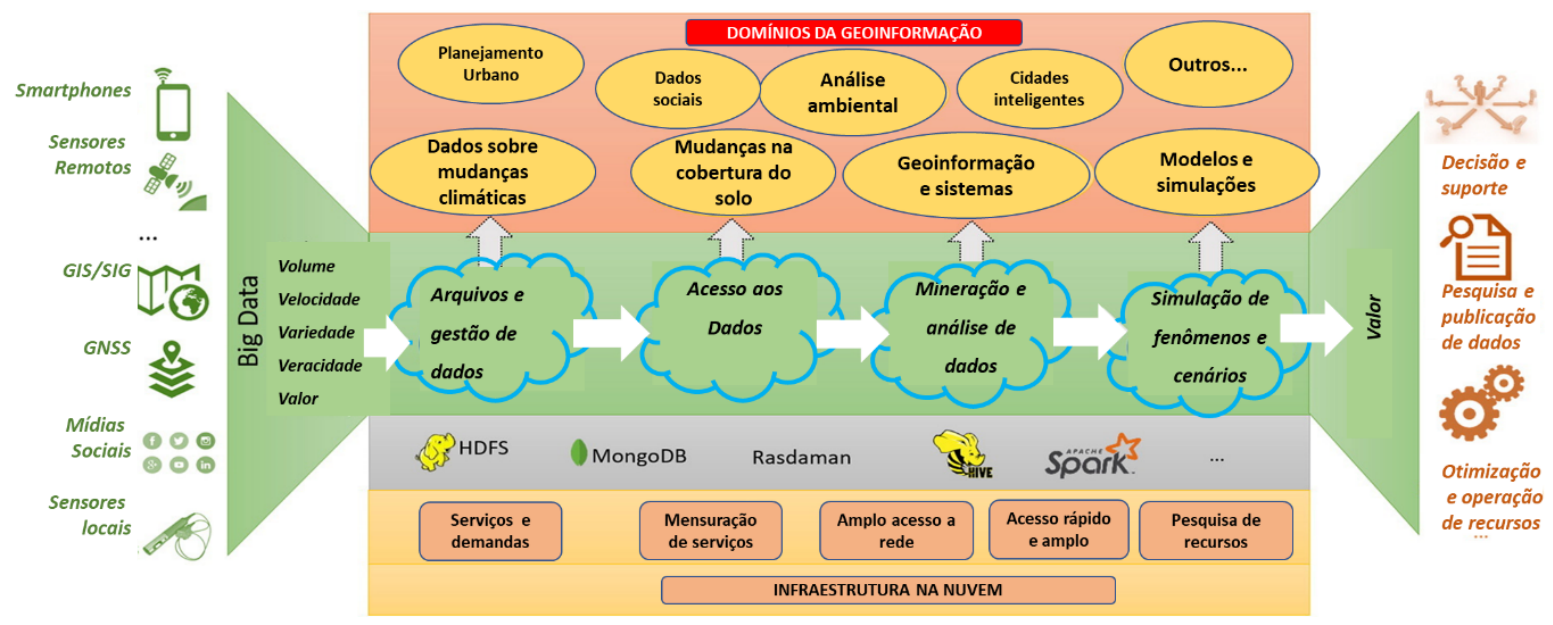

Figura 1. Cloud Computing fornece suporte crítico para o processamento de Big Data para endereçar os $4 \mathrm{Vs}$ para obter valor para melhor suporte de decisão, pesquisa e operações para vários domínios geoespaciais. (Tradução livre). Fonte: adaptado de Yang et al. [2].

Essa combinação de Big Data e Cloud Computing permite a geração de análises com softwares sem a necessidade de instalação local e baixo custo. O potencial de hardware para o processamento dos dados também não é uma preocupação elementar nessa infraestrutura, uma vez que o processamento ocorre na nuvem. Outras vantagens podem ser mencionadas:

- não é preciso pagar por uma licença de software (quando há gasto com aplicativos, geralmente é apenas pelo tempo de uso);

- não há necessidade de atualizar os softwares manualmente ou comprar uma versão mais nova;

- não há necessidade de hardware e sistema operacional mais atualizados;

- compartilhamento facilitado pela nuvem;

- diminuição da necessidade de manutenção;

- dados que podem ser acessados de qualquer lugar com acesso à internet;

Já entre as desvantagens, a principal está ligada ao acesso à internet, caso o acesso seja perdido, o usuário está incapacitado de continuar com sua atividade; bem como se a internet não estiver boa e for preciso velocidade no processamento, isso pode ser prejudicado; como todos os serviços, ele tem um preço; e por último a segurança na nuvem que pode ser prejudicada por inúmeros fatores, entre eles o acesso aos usuários e suas permissões, isso se dá porque os dados ficam online todo o tempo.

No presente capítulo de livro objetiva apresentar as plataformas de mapeamento e análise de dados espaciais em ambiente web, destacando vantagens e características potenciais para o uso em pesquisas e ensino remoto pela Geografia, frente às necessidades atuais de distanciamento social.

\section{Materiais e Métodos}

A metodologia deste capítulo se baseia em apresentar uma breve e objetiva revisão teórica sobre conceitos chaves e em seguida, destacar as plataformas web de análise e mapeamento espacial de dados, destacando características: vantagens e potencialidades. 


\section{Resultados e Reflexões}

\subsection{Falando sobre Big Data e Cloud Computer...}

É perceptível o crescente volume de dados que a sociedade atual gera e disponibiliza a cada segundo. Viana [3] nos aponta que "apesar da humanidade produzir e disseminar informação muito antes das tecnologias atuais surgirem, é a partir da Máquina de Turing, (vale dizer, os computadores, desenvolvida pelo professor de matemática teórica e aplicada Alan Turing durante a Segunda Guerra Mundial (1936), hoje reconhecido como o pai da Ciência da Computação), que se iniciam as primeiras preocupações com os dados, seu armazenamento, sua quantidade, seu processamento e a exploração destes".

Segundo Yang et al. [2] existe atualmente o aumento da produção de dados, nunca se gerou tantos dados como agora principalmente por causa da internet, a sociedade cria a todo momento dados em redes sociais ou aplicativos, isso gerou um crescimento do número das plataformas de computação em nuvem para facilitar o uso desse grande volume e velocidade de dados.

Yang et al. [2] aponta que "o Big Data surge com novas oportunidades de pesquisa, desenvolvimento, inovação e negócios. Caracteriza-se pelos chamados quatro Vs: volume, velocidade, e variedade e pode trazer valor significativo por meio do processamento de Big Data. A transformação dos 4 Vs do Big Data no $5^{\circ}$ (valor) é um grande desafio para a capacidade de processamento." Não existe uma única definição para o conceito de Big Data mas todos giram em torno dos 5V's: Volume; Velocidade; Variedade; Veracidade; e Valor. Além disso, geralmente as soluções que são produzidas a partir desse conceito, normalmente englobam a complexidade, natureza e crescimento dos dados.

A computação em nuvens se apresenta como a evolução do uso de dados, por usuários de forma simultânea, por meio de dispositivos conectados à internet. Nesse caso não é necessário instalar qualquer aplicativo, pois a execução dos aplicativos e armazenamento de dados é de responsabilidade de quem oferta o serviço [4].

Os modelos de serviços definidos pelo NIST (National Institute of Standards and Technology) são: Software as a Service (SaaS), Platform as a Service (Paas) e Infrastructure as a Service (laaS) (Figura 2). Existem vários outros modelos mencionados na literatura, mas os principais e mais usados são os citados pelo NIST.

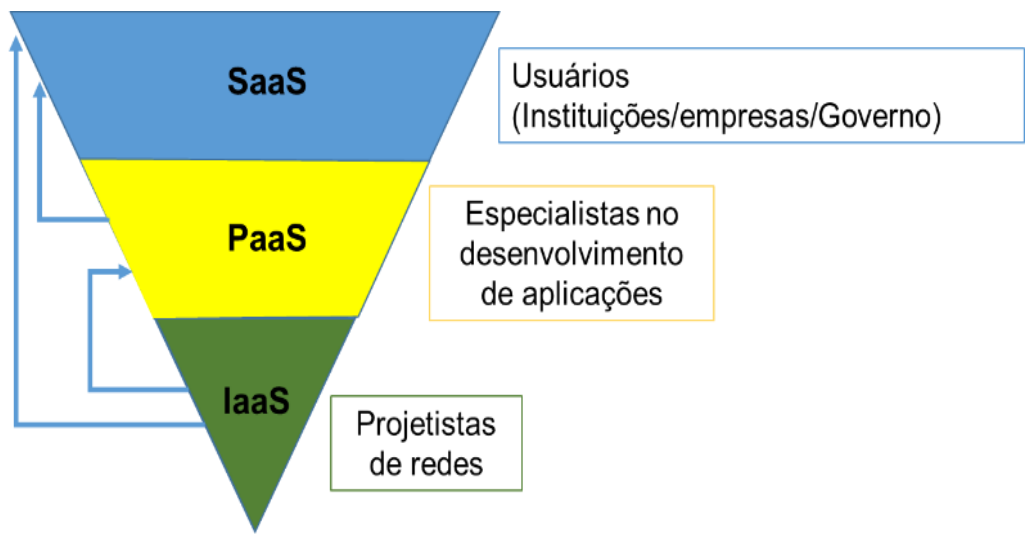

Figura 2. Modelos de serviços na nuvem. Fonte: elaborado pelo autor baseado em Medeiros [5]. 
Segundo Medeiros [5] "O modelo Software as a Service (SaaS) é usado em aplicações com acesso simultâneo por várias pessoas ao mesmo tempo, ou seja, é um modelo de computação em nuvem que é oferecido em forma de serviço, no qual se paga pela hospedagem de softwares que irão rodar remotamente, como por exemplo, o Dropbox e o Google Apps." É possível acessar as aplicações sem a necessidade de instalação de aplicativos. Essa configuração é uma forma de processamento alternativo ao processamento local. Essa camada de aplicações é a que agrega muito mais instituições e usuários, fornecendo serviços e produtos de apoio para análise de dados. Os usuários não são especialistas e não detém conhecimento técnico sobre programação ou manipulação de dados.

O modelo Platform as a Service (PaaS) fornece uma plataforma de softwares onde os usuários podem testar ou desenvolver aplicações de interesse. Para Rountree e Castrillo [6] trata-se de uma plataforma utilizada para o desenvolvimento. Dependendo do provedor, a plataforma pode ser completa incluindo um servidor Web e bibliotecas de desenvolvimento. O Windows Azure e o Google App Engine e o Cloud Foundry (Livre) são os principais representantes. Os especialistas nesse grupo agregam os profissionais dedicados ao desenvolvimento de aplicações para análise e tratamento de dados. O conhecimento exigido para a atuação nessa hierarquia exige um aprofundamento, (no caso de dados espaciais e mapeamento), sobre cartografia, geoinformação e linguagem de programação.

E finalmente, segundo Medeiros [5] o Infrastructure as a Service (laaS) "limita-se ao fornecimento de Infraestrutura para seus usuários, com a finalidade de dar maior liberdade através da Virtualização. Os usuários têm pleno controle sobre suas máquinas virtuais, armazenamento e aplicativos instalados, como, por exemplo, a Amazon Web services." Esse grupo de especialistas integra os especialistas em rede e Tecnologia da Informação - TI.

Em resumo o mapeamento de dados na nuvem se divide em 3 categorias: 1. laaS (Infraestrutura como serviço); 2. PaaS (Plataforma como Serviço); 3. SaaS (Software as a Service), por exemplo, o CARTO. Especificamente na terceira estrutura, em função da natureza dos dados e geoinformação, muitas vezes há a necessidade de adequações. Então nesse caso é comum detectar que o Software as a Service (SaaS) suporta outros três modelos de serviço: GaaS (SIG como serviço); AaaS (Aplicações como Serviço); e laaS (Imagens como Serviço) como consta na Figura 3.

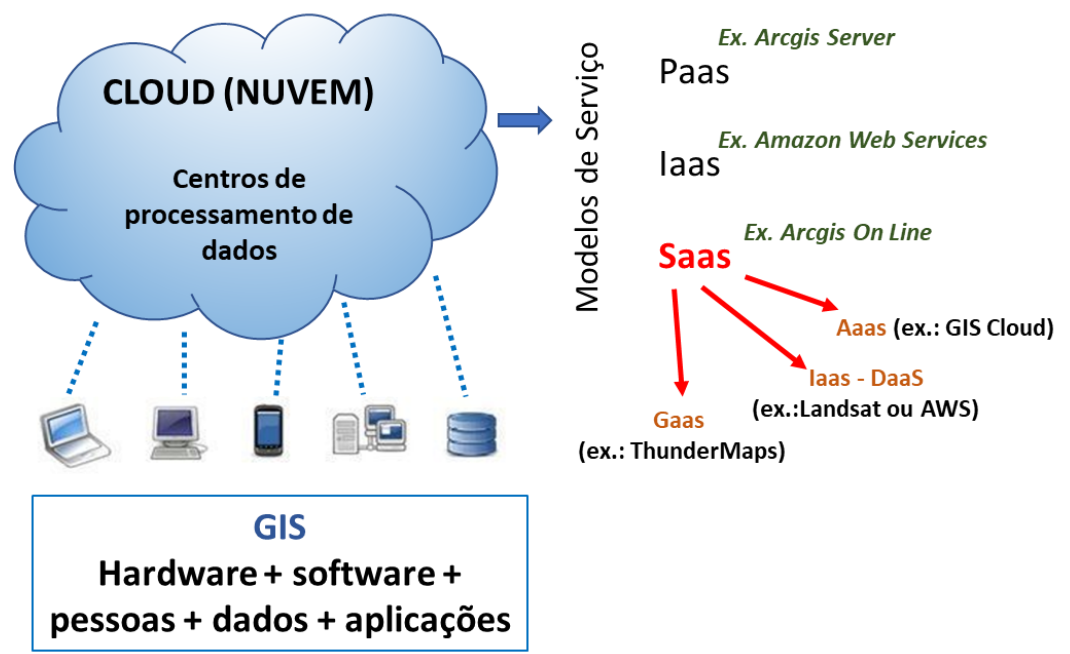

Figura 3. Modelos de serviços na nuvem para dados GIS. Fontes: Imagem obtida em https://www.gislounge.com/ e http://mappinggis.com/2017/01/10-aplicaciones-gis-enla-nube-para-publicar-mapas-en-la-web/ [7]. 


\subsection{Falando sobre mapeamento e GIS WEB, WEBGIS, GIS na Nuvem ou GIS Cloud.}

A área de geoinformação, tradicionalmente utiliza ferramentas desktop, mas devido às limitações como dificuldade de compartilhamento e acesso, cada vez mais se observa o uso de plataformas web para processamento ou armazenamento de dados. 0 mapeamento de dados na web é uma alternativa crescente e cada vez mais presente nas atividades da sociedade. Observa-se com relativa frequência mapas sobre trânsito em tempo real, chuvas em tempo real, acompanhamento de deslocamentos para pedidos de comida delivery, ocorrências médicas e mais atualmente volume de vacinação em massa, em virtude da pandemia da Covid19. Há uma corrida para liderar e apontar os melhores caminhos, plataformas e sistemas. No entanto, há ainda uma necessidade de ampliar o entendimento sobre como funcionam essas plataformas e quais as possibilidades de análise.

O termo WebSIG considera as aplicações que compartilham e integram dados espaciais para os usuários interessados por meio de um navegador na Web, apenas com a disponibilização de um browser de internet, sem que haja a necessidade de instalação de qualquer software. O conceito de "GIS na nuvem ou GIS Cloud" da mesma forma, refere-se ao uso de serviços e aplicativos GIS na Internet, com acesso de usuários a partir de um navegador da Web sem instalar qualquer software no computador utilizado. Essa ferramenta permite usar os aplicativos GIS na nuvem para publicação de mapas, dados e análises na web.

Observa-se uma melhoria no desenvolvimento dos aplicativos na nuvem para dados espaciais em função da melhoria na análise, edição e visualização de resultados. Com isso observa-se o potencial de uso em diversos setores e uma importante alternativa ao SIG de desktop tradicional.

Diante do exposto é importante observar e considerar pontos chaves para a análise dessas plataformas tais como: custos, características gerais e básicas, principais funcionalidades, popularidade, possibilidades de visualização dos dados e desempenho geral das plataformas. Tendo em vista esses critérios de análise foram avaliadas as seguintes plataformas: ArcGIS Online, Carto, Mango Map, Mapbox, Gis Cloud e Google Earth Engine. Essas plataformas são opções importantes para facilitar ou complementar o ensino e a pesquisa na modalidade remota, que é um desafio enfrentado por pesquisadores e docentes durante o curso da pandemia de COVID19.

\subsection{Apresentação geral das plataformas para a geração de mapas na web}

\subsubsection{ArcGIS Online}

O ArcGIS Online é uma plataforma web que faz parte da Esri e permite conectar pessoas, locais e dados utilizando mapas interativos. Trata-se de um serviço na nuvem da Esri com muitas funcionalidades e ferramentas de análise intuitivas que se conectam a outras plataformas de dados, ou mesmo aplicativos variados. O ArcGIS online pode ter um update para uma interface com mais recursos se houver um investimento financeiro.

Seu Software é baseado na nuvem, tem serviço (SaaS), com isso o ArcGis Online pode ser empregado em qualquer lugar, em qualquer instante. A Figura 4 destaca a janela inicial da plataforma e a Figura 5 a estrutura base de funcionamento 


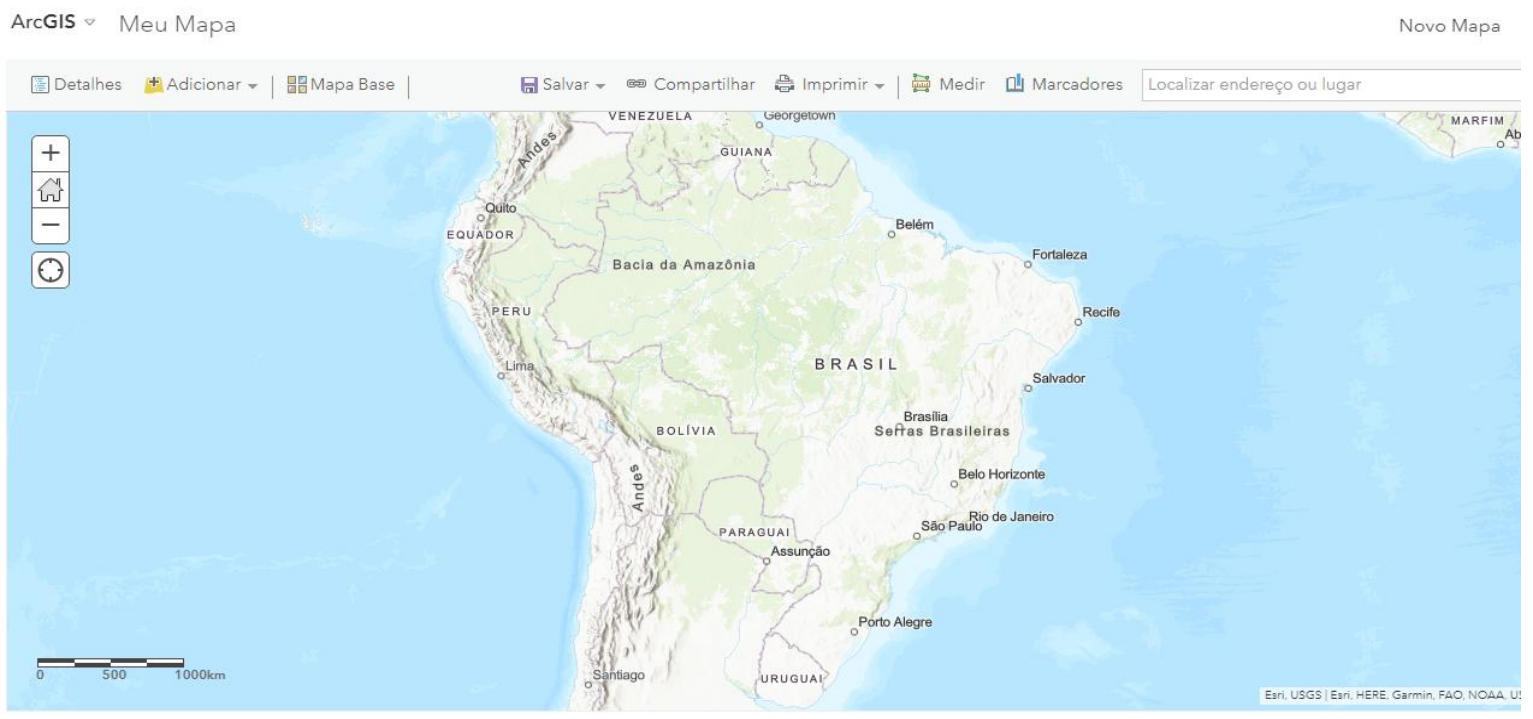

Figura 4. Janela inicial do ArcGis Online.

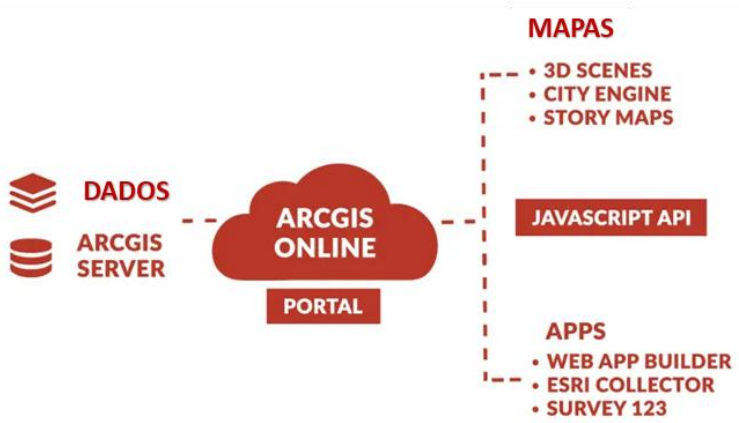

Figura 5. Estrutura de funcionamento do ArcGis Online.

Fonte: https://gisgeography.com/web-mapping/ [8]

\subsubsection{Carto}

Os serviços de Web Postgres + PostGIS são os alicerces da plataforma Carto, o que permite controlar um banco de dados por meio de gerenciamento direto. Essas ferramentas são boas pois permitem ao usuário trabalhar e utilizar a plataforma sem a necessidade de configurações, backups ou atualizações rotineiras. E uma ferramenta robusta pensada para usuários com algum conhecimento de programação, porém aqueles interessados em adotar seu uso, mas que não tenham conhecimento de programação, podem se beneficiar de algumas funcionalidades já implementadas. A Figura 6 destaca um exemplo de aplicação do Carto e a Figura 7 sua estrutura de funcionamento. 


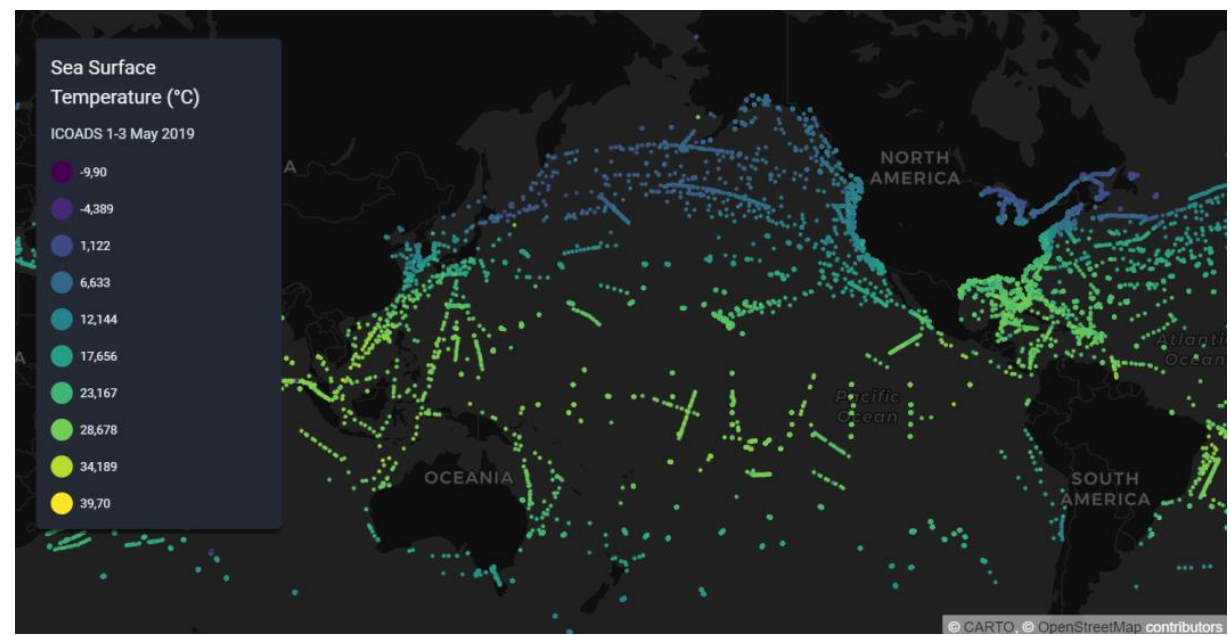

Figura 6. Plataforma Carto e um exemplo de mapeamento da temperatura superficial dos oceanos em maio de 2019 (elaboração do autor utilizando a plataforma).

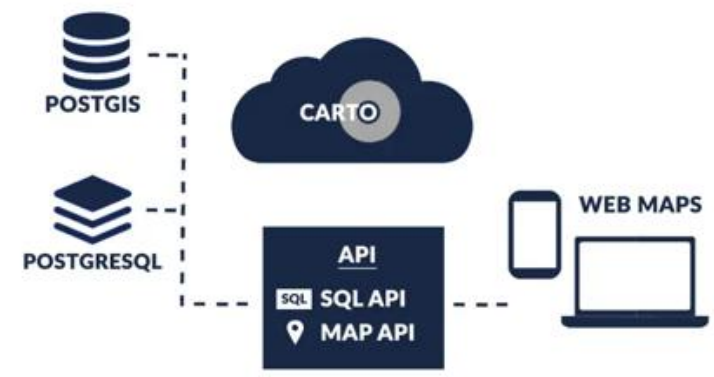

Figura 7. Estrutura de funcionamento do Carto.

Fonte: https://gisgeography.com/web-mapping/ [8].

\subsubsection{Mapbox}

Mapbox foi pensado para uso por programadores, como o Carto, no entanto, da mesma forma, há muitas aplicações implementadas para uso por não especialistas em programação. Uma das vantagens é a ótima interface visual de acessos aos dados. Trabalha com bibliotecas JavaScript e muitos plug-ins para estender as funcionalidades. A Figura 8 destaca um exemplo de aplicação do Mapbox.

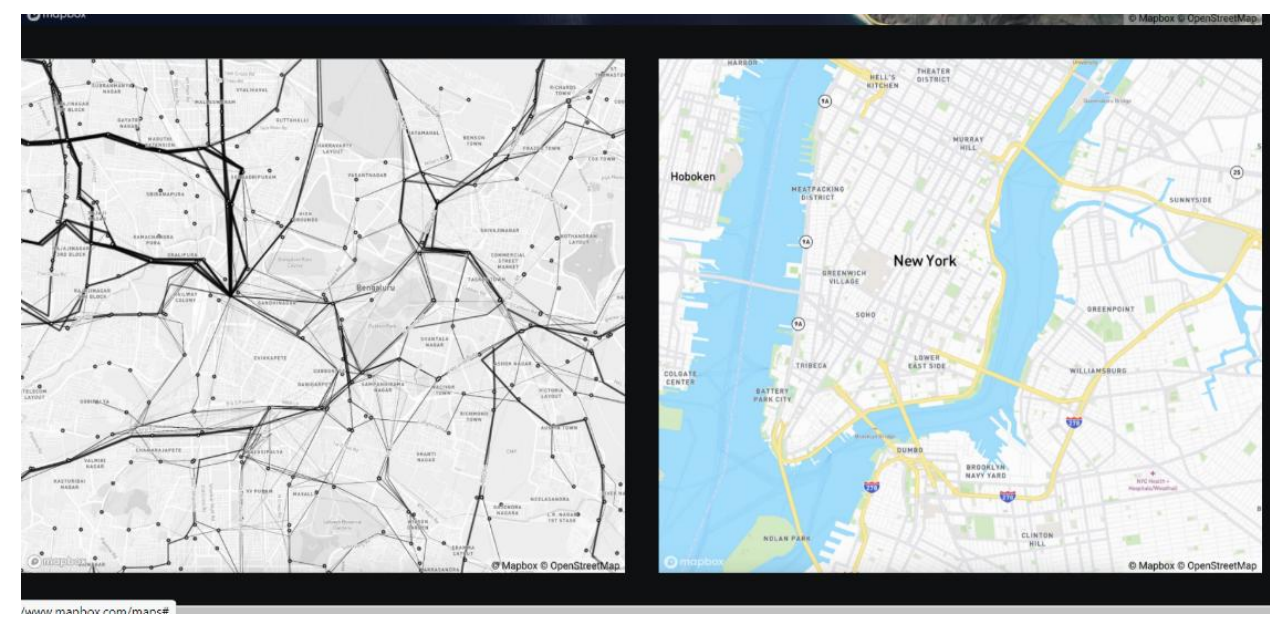

Figura 8. Plataforma Mapbox e possíveis aplicações (elaboração do autor utilizando a plataforma). 


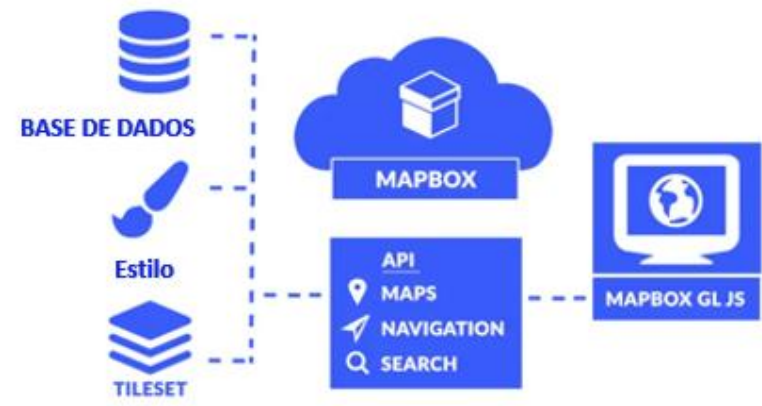

Figura 9. Estrutura de funcionamento do Mapbox.

Fonte: https://gisgeography.com/web-mapping/ [8].

\subsubsection{Mango Map}

De todas as plataformas mencionadas até aqui a Mango Map possui a mais fácil interface para a publicação de dados. Há uma baixa complexidade o que facilita o acesso por qualquer usuário. Não há a necessidade de programar para começar a utilizar a plataforma.

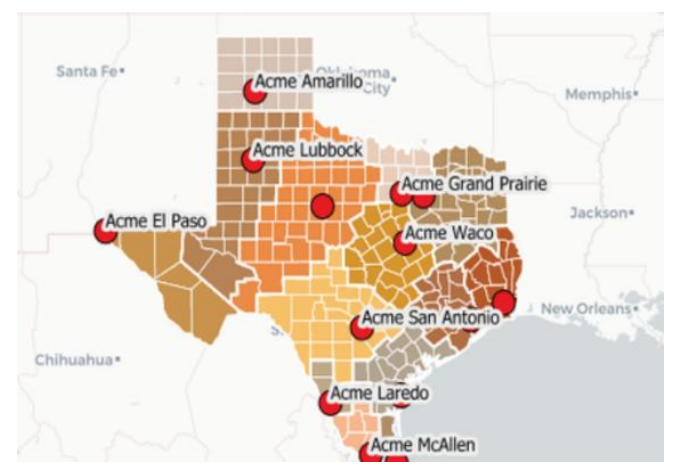

Figura 10. Mango Map ferramenta (elaboração do autor utilizando a plataforma).

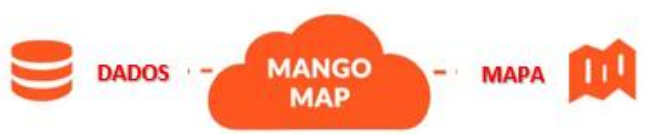

Figura 11. - Estrutura de funcionamento do Mango Map.

Fonte: https://gisgeography.com/web-mapping/ [8].

\subsubsection{GIS Cloud}

Segundo Kholoshyn et al. [9] as principais vantagens do GIS Cloud são: acesso ininterrupto às informações desde que esteja em qualquer computador conectado a internet; não há necessidade de instalar um pacote GIS em seu computador; armazenamento de dados pessoais na nuvem: pacotes de mapas, camadas, dados tabulares (atributos); acesso aos recursos espaciais preparado e publicado por outros 
usuários (vários mapas, desde físicos e geográficos até econômicos e políticos, imagens de satélite, mapas históricos, mapas interativos, entre outros); possibilidade de criação de mapas interativos, bem como criação baseada na web aplicativos baseados em modelos pré-fabricados sem o uso de ferramentas de programação; facilidade de cooperação integrada entre os usuários dentro de uma organização educacional.

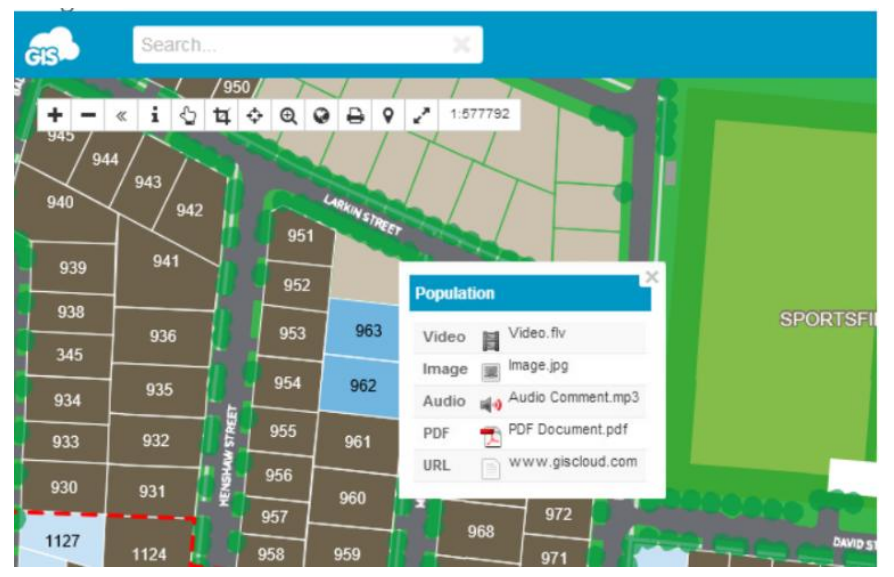

Figura 12. GIS Cloud ferramenta (elaboração do autor utilizando a plataforma).

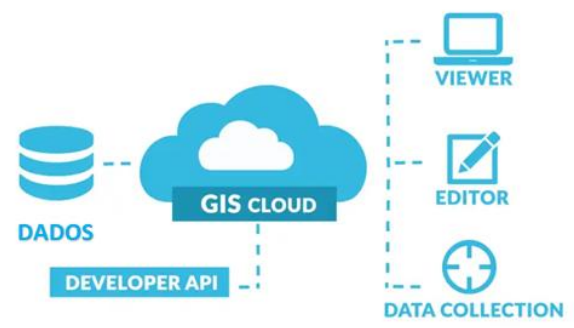

Figura 13. Estrutura de funcionamento do GIS Cloud

Fonte: https://gisgeography.com/web-mapping/ [8].

\subsubsection{Google Earth Engine}

O Google Earth Engine (GEE) é uma plataforma para análise científica e visualização de conjuntos de dados geoespaciais para uso acadêmico, empresarial, governamental e sem fins lucrativos. A plataforma hospeda imagens de satélite e as armazena em um arquivo de dados público que inclui imagens históricas da Terra, uma sequência temporal de mais de 40 anos de imagens, coletadas e disponibilizadas diariamente, para mineração de dados em escala global. O catálogo de dados é combinado com poder de computação escalonável apoiado pelos centros de dados do Google e interfaces de programação de aplicações que permitem implementar perfeitamente seus fluxos de trabalho geoespaciais existentes, permitindo análise e visualização de grandes áreas. A Figura 14 destaca as plataformas do Google Earth Engine. 


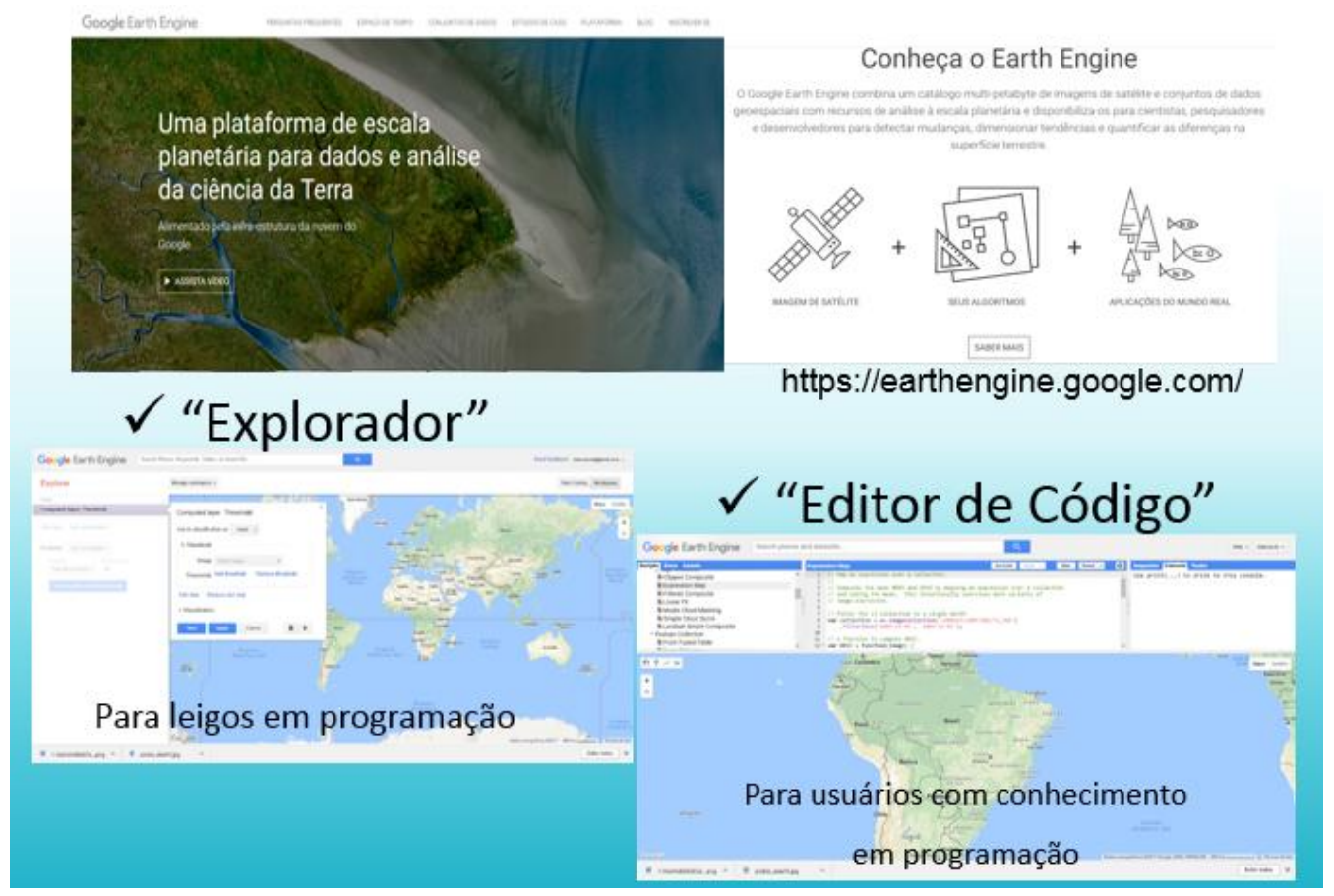

Figura 14. Plataformas de processamento de dados GEE (elaboração do autor utilizando a plataforma).

Vasconcelos [10] relata que a ideia de criar uma ferramenta gratuita com o potencial do GEE surgiu em 2008, quando a Google esteve no Brasil para lançar um projeto. Observou-se então que existia uma necessidade de criar esse tipo de plataforma, após muitas reuniões no Brasil e nos Estados Unidos, envolvendo os esforços de vários pesquisadores, em 2009 durante a Convenção do Clima em Copenhague, foi apresentada uma versão beta do que hoje é o Google Earth Engine.

Sobre a plataforma GEE conseguimos encontrar no próprio site as seguintes informações: é uma plataforma de computação que permite aos usuários executar análises geoespaciais na infraestrutura do Google. O usuário precisa ter uma conta no Google para fazer login e ter acesso ao Earth Engine. Existem várias maneiras de interagir com a plataforma. O Editor de código do Earth Engine é um IDE (ambiente de desenvolvimento integrado) baseado na web para a API (interface de programação de aplicações) JavaScript do Earth Engine. Os recursos do Editor de código são projetados para tornar rápido e fácil o desenvolvimento de fluxos de trabalho geoespaciais complexos. O Explorer é um aplicativo da web leve para explorar nosso catálogo de dados e executar análises simples, para quem não é desenvolvedor. Ele permite que qualquer pessoa visualize os dados do catálogo público de dados. Os usuários conectados ao Google Earth Engine podem importar dados, executar análises simples, salvar e exportar os resultados. As bibliotecas encontradas na plataforma fornecem conjuntos de funções Python e JavaScript em torno da interface de programação de aplicações da web [11].

Segundo Gorelick et al. [11] entre os conjuntos de dados disponíveis estão todo o catálogo Landsat EROS (USGS/NASA), vários conjuntos de dados MODIS, Sentinel-1, NAIP, dados de precipitação, dados de temperatura da superfície do mar, dados climáticos CHIRPS e dados de elevação. Os usuários também podem fazer upload de 
seus próprios dados para análise no Earth Engine, com controle total sobre o acesso, o GEE permite que você envie seus próprios dados raster e vetoriais (por exemplo, arquivos GeoTIFF ou Shapes) para análise. Além da possibilidade de compartilhar e publicar os resultados e scripts de análise diretamente com outros usuários ou simplesmente fazer o download e compartilhar como faria com qualquer outro dado.

Um estudo recente, Amani et al. [12], que visa explorar de forma abrangente diferentes aspectos da plataforma e várias aplicações do GEE, incluindo seus conjuntos de dados, funções, vantagens e limitações, constatou que a plataforma do Google Earth Engine tem sido utilizado para uma grande variedade de aplicações, como planejamento urbano, classificação de uso e cobertura da terra, hidrologia, agricultura, vegetação, análises climáticas e processamento de imagens, além disso confirmou um grande aumento no volume de publicações nos últimos anos e há a expectativa que a plataforma seja cada vez mais utilizada para resolver desafios de processamento de Big Data.

O Google Earth Engine é uma poderosa plataforma com diversos recursos e qualidades, porém também possui suas limitações tanto na infraestrutura de nuvem, como em API, dados e funções.

O primeiro ponto de destaque serão as restrições, afinal são relativamente pequenas, mas é muito importante o usuário estar ciente delas: mesmo os dados sendo privados na conta do usuário, eles são armazenados nos servidores de uma empresa; a análise de imagens é restrita às ferramentas da API; alguns métodos de pré-processamento como correção atmosférica não são implementados; a classificação de imagens pode ser melhorada pela análise de imagens baseada em objetos, o algoritmo de segmentação para esse fim não é eficiente; ao longo do processo se precisar baixar dados para análise posterior pode haver demora pelo tamanho do mapa ou limitações da internet; poucos algoritmos de classificação e regressão, como CART, RF e SVM; limitações das aplicações de SAR por motivo de incompatibilidade com a infraestrutura; limitações com relação ao uso de algoritmos complexos de aprendizado de máquina; número limitado de recursos ou amostras de treinamento nos métodos de classificação.

As vantagens do GEE são incontáveis, entre elas existem as já descritas vantagens da Computação em Nuvem, como não precisar baixar e gerenciar dados localmente; com as operações sendo realizadas em paralelo nas CPUs e GPUs do Google, podendo processar, no caso específico do Earth Engine, petabytes de dados de sensoriamento remoto em grandes escalas geográficas e em longas coberturas temporais, além da possibilidade de fazer upload e compartilhar seus próprios conjuntos de dados; existem também recursos de filtragem e classificação rápidos permitindo que os usuários selecionem seus dados desejados entre milhões de imagens com base em várias especificações espaço-temporais.

GEE é combinado com uma poderosa interface de programação baseada na web. Os usuários acessam facilmente os dados de sensoriamento remoto arquivados por meio da API (interface de programação de aplicações) JavaScript e Python e cada uma delas tem as suas facilidades. O conceito simples de usar essas duas APIs permite que os usuários se concentrem na lógica da seleção de dados e no fluxo de trabalho programável, um editor de código online também está disponível para escrever scripts, depurá-los e ver os resultados logo após a compilação. A biblioteca do Google Earth Engine também é uma de suas vantagens, existe nela inúmeras funções e algoritmos como de processamento de imagem e vetorial, aprendizado de máquina, análise geométrica que permite o usuário elaborar seu projeto, além de alguns tutoriais de códigos e métodos de importação e exportação de dados facilitados pelo GEE. 
Outra vantagem diz respeito aos dados, um vasto conjunto de dados de sensoriamento remoto, meteorológico, geofísico, incluindo os mais de 40 anos de imagens Landsat, muito importante para análise temporal. Existem os dados e metadados originais na plataforma, mas as resoluções são gerenciadas por ela para ser eficiente em vários níveis de zoom. Existem dados pré-processados e dados brutos, bem como produtos derivados, índices espectrais populares (por exemplo, NDVI) já estão calculados. Além de todos esses pontos positivos expostos até aqui, na página inicial, o usuário consegue ter acesso a estudos de caso mundialmente famosos e ver do que essa ferramenta é capaz.

Para exemplificar as inúmeras funcionalidades da plataforma, na Figura 15 podemos ver uma classificação, onde a seleção das amostras (pelo usuário) feita em uma imagem da própria base de dados e utilizando um algoritmo (CART) foi feita quase instantaneamente.

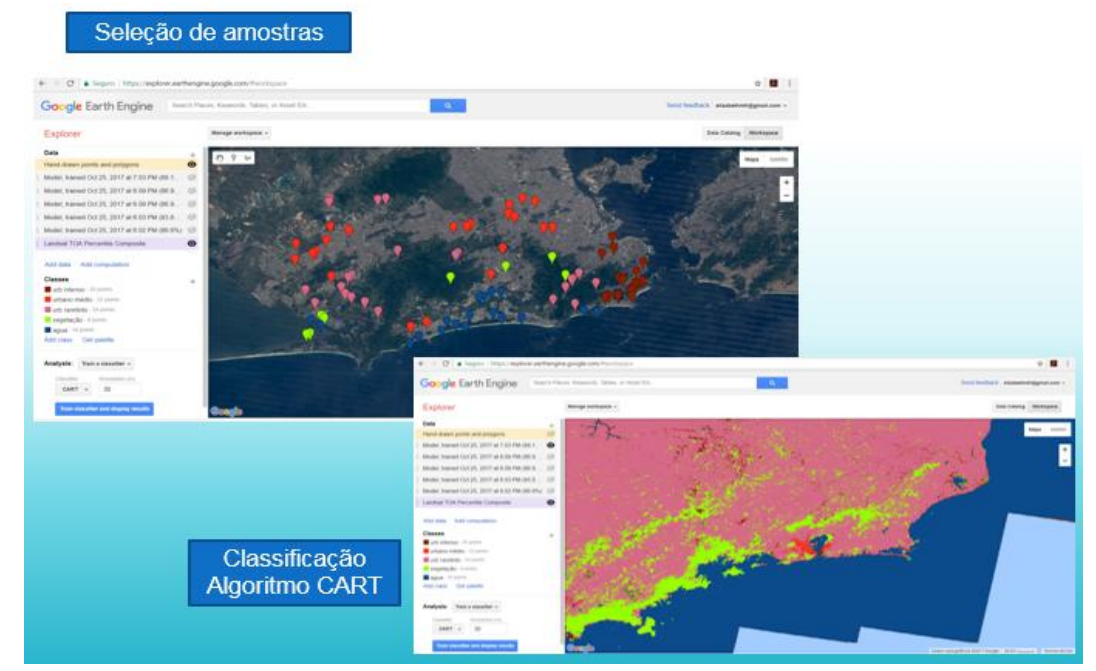

Figura 15. Classificação simples feita no GEE (elaboração do autor utilizando a plataforma).

\subsection{Potenciais e usos para pesquisa e ensino}

É importante destacar os potenciais usos das plataformas nas pesquisas e no ensino remoto da Geografia.

\subsubsection{Potenciais ações e usos das plataformas de mapeamento WEB}

A Tabela 1 destaca os principais potenciais de uso das plataformas apresentadas, ressaltando o nível de dificuldade para acesso e uso dos dados. Foram classificadas em 3 níveis de dificuldade (baixa, média e alta) com possibilidade de uso em atividades de pesquisa e ensino.

Como principais desafios e perspectivas para o uso dessas plataformas pode-se citar: o pouco conhecimento sobre o acesso e uso as versões; a delimitação dos melhores insumos (imagens, dados e modelos) para obtenção de informação relevante para Geografia; e o preparo e formação de futuros geógrafos com conhecimento suficiente para a implementação de ferramentas de análise e o custo de acesso ao potencial completo dessas ferramentas. 
Tabela 1. Potencial de uso e níveis de dificuldade das plataformas WEB.

\begin{tabular}{|r|l|l|l|l|l|}
\hline AÇÕES & $\begin{array}{c}\text { ARCGIS } \\
\text { ON } \\
\text { LINE }\end{array}$ & CARTO & $\begin{array}{c}\text { MAP } \\
\text { BOX }\end{array}$ & $\begin{array}{c}\text { MANGO } \\
\text { MAP }\end{array}$ & $\begin{array}{c}\text { GIS } \\
\text { CLOUD }\end{array}$ \\
\hline $\begin{array}{r}\text { Criação e edição de } \\
\text { dados }\end{array}$ & & & & & \\
\hline $\begin{array}{r}\text { Publicação de mapas e } \\
\text { dados }\end{array}$ & & & & & \\
\hline $\begin{array}{r}\text { Ferramentas para } \\
\text { Análise Espacial }\end{array}$ & & & & & \\
\hline $\begin{array}{r}\text { Introdução de dados } \\
\text { externos }\end{array}$ & & & & & \\
\hline
\end{tabular}

Baixo

Médio

Alto

\subsubsection{Google Earth Engine}

A Tabela 2 destaca os principais potenciais de uso do GEE ressaltando as temáticas beneficiadas e insumos.

Para o completo entendimento sobre a manipulação da plataforma Google Engine Explorer recomenda-se a leitura dos manuais disponíveis no website da Google. Do conjunto de ferramentas pré-definidas e disponíveis cabe destacar: o conjunto de imagens DMSP-OLS Nighttime Lights Time Series Version 4, os mosaicos anuais do Landsat TOA Percentile Composite; a ferramenta para classificação e seleção de amostras Hand-drawn points and polygons e os modelos de relevo SRTM Digital Elevation Data com resolução de 30m.

O conjunto de imagens DMSP-OLS Nighttime Lights Time Series Version 4 representa uma série histórica de imagens noturnas que mensura a cada ano, a quantidade e intensidade de luz emitida durante a noite no globo terrestre. As imagens de satélite possuem uma resolução espacial de $900 \mathrm{~m}^{2}$ e possibilitam uma série de análises espaciais sobre os vetores de expansão urbana. Pode-se trabalhar com a delimitação de áreas urbanas, monitoramento e expansão e ainda aspectos particulares como a questão energética e política.

Os mosaicos anuais do Landsat TOA Percentile Composite em conjunto com a ferramenta para classificação e seleção de amostras Hand-drawn points and polygons pode permitir a execução de várias classificações de uso e cobertura do solo.

Também estão disponíveis dados de relevo, sensores com aplicação para análise climática (dados do satélite NOAA), e outros com maior resolução espacial (imagens do satélite Sentinel).

Como principais desafios e perspectivas pode-se citar: a criação de métodos visando o monitoramento de desmatamento em tempo real de forma automática; a delimitação dos melhores insumos (imagens e modelos) para obtenção de informação relevante para Geografia; e o preparo e formação de futuros geógrafos com conhecimento suficiente para a implementação de ferramentas de análise. É importante destacar dentre as iniciativas nacionais com o uso da plataforma Google Earth Engine, a construção do portal MapBiomas. Trata-se de um projeto que visa produzir mapas anuais da cobertura e uso do solo no Brasil, desde 1985 até os dias atuais. Os mapas irão disponibilizar informações e estatísticas sobre a cobertura e uso do solo para cada 
ano, em diversas escalas (município, estado, bioma), e também as transições de um ano para o outro. A partir dos mapas produzidos será possível identificar as transformações do uso da terra em áreas com floresta, pastagens, agricultura, reflorestamento e áreas urbanas, dentre outros temas, em todo o território nacional.

Tabela 2. Potencial de uso do GEE e temáticas para a Geografia.

\begin{tabular}{|c|c|c|c|c|c|c|c|c|c|c|}
\hline $\begin{array}{c}\text { Temáticas de estudo } \\
\text { (Geografia) }\end{array}$ & $\begin{array}{l}\text { Geografia Urbana e População } \\
\text { Geografia Agrária } \\
\text { Geografia Regional } \\
\text { Geografia Política e Econômica } \\
\text { Geografia Fisica } \\
\text { Geologia, Geomorfologia } \\
\text { Pedologia e Recursos Hídricos } \\
\text { Climatologia e Biogeografia } \\
\text { Descrição da aplicação }\end{array}$ & 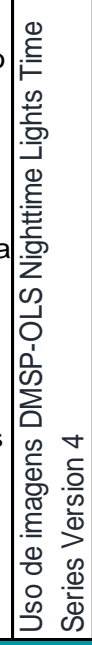 & 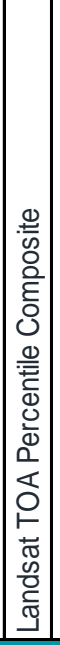 & 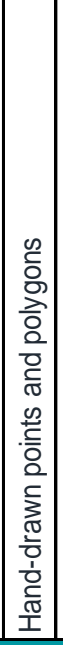 & 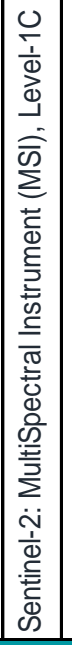 & 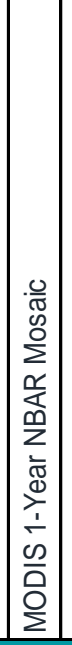 & 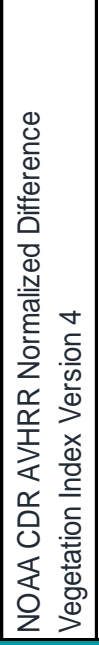 & 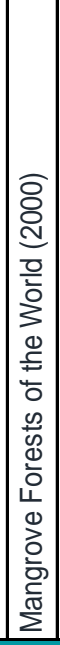 & 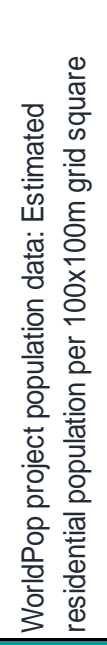 & 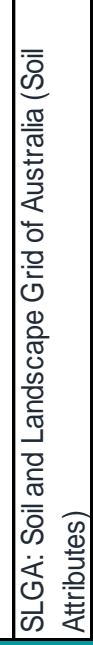 \\
\hline $\begin{array}{r}\text { Processo de } \\
\text { Globalização e a } \\
\text { constituição da rede } \\
\text { alobal de cidades } \\
\end{array}$ & Delimitação e monitoramento & & & & & & & & & \\
\hline $\begin{array}{r}\text { O processo de } \\
\text { urbanização e recorte } \\
\text { espacial }\end{array}$ & Delimitação e monitoramento & & & & & & & & & \\
\hline $\begin{array}{r}\text { Organização do espaço } \\
\text { interno das cidades e } \\
\text { segregação social } \\
\text { urbana }\end{array}$ & Delimitação e análise espacial & & & & & & & & & \\
\hline mobilidade urbana & Definição de fluxos/Rede & & (a) & & $\bar{z}$ & & & & & \\
\hline $\begin{array}{r}\text { Vulnerabilidade e risco } \\
\text { ambiental } \\
\end{array}$ & Criação de indicadores & & & & & & & & & \\
\hline Planejamento e Gestão & dentificação e mensuração de áreas & & & & & & & - & & \\
\hline $\begin{array}{l}\text { Geomorfologia: } \\
\text { Processos exógenos e } \\
\text { endógenos, formas de } \\
\text { relevo e/ou pedologia }\end{array}$ & Delimitação e análise espacial & & & & & & & & & \\
\hline $\begin{array}{r}\text { As dimensões espaciais } \\
\text { do poder; Poder, } \\
\text { Território e } \\
\text { Territorialidade; Limites e } \\
\text { fronteiras }\end{array}$ & Reconhecimento de padrão espacial & & & & & & & & & \\
\hline $\begin{array}{r}\text { A produção e consumo } \\
\text { do espaço }\end{array}$ & Reconhecimento de padrão espacial & & & & & & & & & \\
\hline
\end{tabular}

\section{Conclusão}

Com as mudanças que estão acontecendo nos últimos tempos, a busca por novas demandas tecnológicas devido a forçada adaptação ao meio online, remoto e digital por ocorrência da pandemia de Covid-19, fica notável a necessidade dos profissionais por demandas de ferramentas inovadoras e de como usá-las, o desafio é entender 
como elas podem ser aplicadas ou quais seus potenciais, tendo em vista as diferentes nuances dos contextos sociais e econômicos.

Considerando o cenário ideal para um professor em que todos os alunos têm acesso a um computador com internet, pode-se dizer que nesse contexto, com a nova realidade, as plataformas se mostram úteis no que se refere a ensino a distância. Elas são intuitivas para alguém que está familiarizado com os softwares desktops utilizados tradicionalmente, em relação os dados, os usuários podem utilizar os que são disponibilizados pelas plataformas (ArcGIS Online e GEE tem uma base de dados própria, o que garante que que análises sejam feitas sem importar nenhum dado de outras fontes se não houver necessidade), mas se forem dados importados são facilmente incorporados por elas, ambas as plataformas tem facilidade no acesso e são grátis, compartilhar dados com outros usuários também não é difícil.

Todas as plataformas contam com mapas interativos, funções, aplicações, e opções de compartilhamento e pode-se afirmar que essas plataformas podem contribuir para democratização do acesso e propagação desses conhecimentos no período da pandemia da covid-19.

\section{Referências}

[1] Amaral F. Introdução à ciência de dados: mineração de dados e big data. 1aㅡ ed Rio de Janeiro: Alta Books Editora; 2016. 320 p.

[2] Yang C., Yu M., Hu F., Jiang Y., Li Y. Utilizing Cloud Computing to address big geospatial data challenges. Computers, Environment and Urban Systems 2016; 61(B): 120:128. https://doi.org/10.1016/j.compenvurbsys.2016.10.010.

[3] Viana J.M.A. Representação colaborativa de dados científicos: estudo na rede de repositórios de dados científicos do estado de São Paulo. Dissertação de Mestrado. São Paulo: Universidade Federal de São Paulo; 2020.

[4] Fenilli A.T.R., Marchi K.R.C. Computação em nuvem: um futuro presente. Paranavaí, 2011. Disponível em: <https://docplayer.com.br/642983-Computacao-emnuvem-um-futuro-presente.html>. Acesso em: 15 de mar. 2021.

[5] Medeiros M.C.P. Análise da implantação de computação em nuvem: estudo de caso na Alfa Informática.net - Caicó. Currais Novos. Trabalho de Conclusão de Curso. Caicó: Universidade Federal do Rio Grande do Norte; 2015.

[6] Rountree D., Castrillo I. The Basics of Cloud Computing: Understanding the Fundamentals of Cloud Computing in Theory and Practice. Elsevier; 2013. https://doi.org/10.1016/C2012-0-02521-5.

[7] GIS Lounge e MappingGIS. https://www.gislounge.com/ e http://mappinggis.com/2017/01/10-aplicaciones-gis-en-la-nube-para-publicar-mapasen-la-web/. Acesso em: 01 de nov. 2020.

[8] GISGeography. https://gisgeography.com/web-mapping/. Acesso em: 01 de fev. 2021.

[9] Kholoshyn I.V., Bondarenko O.V., Hanchuk O.V., Shmeltser E.O. Cloud ArcGIS Online as an innovative tool for developing geoinformation competence with future geography teachers Ucrânia, 2019. Disponível em: <https://arxiv.org/abs/1909.04388> Acesso em: 15 de mar. 2021. 
[10] Vasconcelos Y. Colaboração do céu. Revista Pesquisa FAPESP 230. São Paulo. p64-69. Abril. 2015. Disponível em: <https://revistapesquisa.fapesp.br/wpcontent/uploads/2015/04/064-069_Google-Engine_230.pdf>. Acesso em: 15 de mar. 2021.

[11] Gorelick N., Hancher M., Dixon M., Ilyushchenko S., Thau D., Moore R. Google Earth Engine: análise geoespacial em escala planetária para todos. Sensoriamento Remoto do Meio Ambiente <https://earthengine.google.com/>.

[12] Amani M., Ghorbanian A., Ahmadi S.A., Kakooei M., Moghimi A., Mohamm S. Google Earth Engine Cloud Computing Platform for Remote Sensing Big Data Applications: A Comprehensive Review. IEEE Journal of Selected Topics in Applied Earth Observations and Remote Sensing 2020; 13:5326-5350. https://doi.org/10.1109/JSTARS.2020.3021052.

\section{Autores}

Haillany Silva de Souza, Sherlyê Francisco de Carvalho, Elizabeth Maria Feitosa da Rocha de Souza*

Departamento de Geografia, Universidade Federal do Rio de Janeiro, Rua Athos da Silveira Ramos 274 - CCMN - Bloco I Sala 12, Rio de Janeiro, Brasil.

*Autor para correspondência: elizabethmfr@gmail.com 